\title{
LEPROSY AND TUBERCULOSIS
}

At the 24th World Conference of the International Union Against Tuberculosis, held in Brussels, 5 to 9 September 1978, a two-hour plenary session was devoted to leprosy. Although a regrettably small proportion of those attending appeared to find the subject of leprosy sufficiently attractive, many doctors from countries of the Third World who are today faced with the 
problems of both leprosy and tuberculosis were eager to avail themselves of the expertise provided by such well-known figures as Drs Michel Lechat, $\mathrm{H}$. Sansarricq, S. G. Browne, S. Pattyn, E. Freerksen and R. J. W. Rees.

The various contributions indicated the similarities between the two diseases, and also the differences. In leprosy, the continued lack of a method of culture of the causative organism on artificial media still hampers investigations, as does the apparent impossibility of matching the excellent controlled clinical trials in tuberculosis that have meant so much in the popularization of precise programmes of multi-drug therapy.

With governments examining, or actually adopting policies of combined attack on both these "tropical" scourges, it behoves those concerned primarily with leprosy to renew their efforts to arrest the disease in patients suffering from multibacillary forms of leprosy and prevent the infection of susceptible contacts. The increasing menace of drug resistance, the investigation of which has owed much to the earlier work on tuberculosis, lends point to many of the views on this theme expressed during this interesting session at the Congress.

At a sectional meeting, some of the microbiological aspects of the two diseases were discussed.

Thanks are due to Dr Annik Rouillon, the dynamic Director of Scientific Activities of the World Conference of the International Union against Tuberculosis for her initiative in suggesting these sessions and for her contacts with the Medical Commission of ILEP. 